\title{
CAPACIDADES ORGANIZACIONALES Y COMPETENCIAS PARA LA DIVERSIFICACIÓN PRODUCTIVA: EL CASO DE ARROZ Y PACÚ EN CHACO, ARGENTINA ${ }^{1}$
}

\author{
Verónica Robert* \\ Nicolás Moncaut** \\ Marianela Perez ${ }^{* * *}$ \\ Gregorio Miranda**** \\ Miguel Alegre* ${ }^{* \star * *}$ \\ Renzo Balbiano $* * * * * *$ \\ Ignacio Ossola ${ }^{* * * * * *}$
}

* Universidad Nacional de San Martín (UNSAM), Argentina y Consejo Nacional de Investigaciones Científicas y Técnicas (CONICET), Argentina.

Contacto: vrobert@gmail.com

** Universidad Nacional de San Martín (UNSAM), Argentina y Consejo Nacional de Investigaciones Científicas y Técnicas (CONICET), Argentina.

Contacto: nmoncaut@gmail.com

*** Escuela de Gobierno de Chaco, Argentina.

Contacto: marianelaperez@escueladegobierno.chaco.gov.ar

**** Escuela de Gobierno de Chaco, Argentina.

Contacto: asistente1@escueladegobierno.chaco.gov.ar

***** Escuela de Gobierno de Chaco, Argentina.

Contacto: miguelalegre@escueladegobierno.chaco.gov.ar

****** Escuela de Gobierno de Chaco, Argentina.

Contacto: renzobalbiano@escueladegobierno.chaco.gov.ar

******* Escuela de Gobierno de Chaco, Argentina.

Contacto: ignacioossola@escueladegobierno.chaco.gov.ar

1 Trabajo realizado en el marco del seminario "Experiencias locales de desarrollos productivos innovadores" de la Escuela de Gobierno de la Provincia del Chaco. Presentado en las X Jornadas nacionales de investigadores en economías regionales", Rosario, Argentina, y XXII Reunión Anual de la RED PYMES MERCOSUR, Montevideo, Uruguay. Agradecemos los comentarios recibidos en dichas ocasiones, así como los realizados por los/as evaluadores/as anónimos/as. Por último, también queremos agradecer a las personas entrevistadas en el curso de la investigación. 
Resumen. En un periodo de apenas cinco años, un productor de arroz del este de la provincia de Chaco (Argentina) llevó a cabo un proceso innovador de diversificación productiva de gran envergadura. A partir de 2010, tras un reclamo ambiental, integró su actividad principal al cultivo y procesamiento de pacú, bajo un esquema de rotación entre ambas producciones. A partir de esto, se desataron cambios de magnitud en la organización que obligaron a coordinar una serie de actividades nuevas y complementarias al nuevo producto (procesamiento, logística y comercialización del nuevo producto, abastecimiento de insumos y requerimientos tecnológicos específicos). Desde la perspectiva de las competencias organizacionales, capacidades y rutinas de la firma, la diversificación requiere el desarrollo de importantes procesos de aprendizaje. Por otra parte, la literatura de pioneros sostiene que las primeras empresas en ingresar a una nueva actividad potencialmente allanan el camino a seguidoras. Este caso permite reconsiderar algunas de las principales hipótesis de ambos conjuntos de literaturas: competencias organizacionales y pioneros; ya que pone de manifiesto la complejidad de los procesos de innovación y diversificación productiva, y sus implicancias tanto al interior de la organización, como en su relación con el entorno.

Palabras Clave: Competencias; Pioneros; Agroindustria. 


\title{
ORGANIZATIONAL CAPABILITIES AND COMPETENCES FOR PRODUCTIVE DIVERSIFICATION: THE CASE OF RICE AND PACÚ IN CHACO, ARGENTINA
}

\begin{abstract}
In just five years, a rice producer in the eastern province of Chaco (Argentina) undertook an innovative process of large-scale production diversification. Since 2010, after an environmental claim, he integrated his main activity to the cultivation and processing of pacú, under a rotation scheme between both productions. Then, great changes were made in the organization of the company, which led to the coordination of a series of new and complementary activities to the new product (processing, logistics and commercialization of the new product, supply of inputs and specific technological requirements). On the one hand, from the perspective of organizational capabilities, skills and routines of the firm, diversification requires the development of important learning processes. On the other hand, the literature of pioneers argues that the first companies to enter in a new activity potentially facilitate the emergence of followers. This case allows us to reconsider some of the main hypotheses of both sets of literatures: organizational competencies and pioneers. It shows the complexity of the processes of innovation and productive diversification and their implications both within the organization and in its relationship with the environment.
\end{abstract}

Keywords: Competences; Pioneers; Agro-industry.

Original recibido el 07/08/2017

Aceptado para su publicación el 11/02/2019

SaberEs. Vol. 11, Núm.1 (2019). 41-64 Sección Artículos 


\section{Introducción}

En un periodo de apenas cinco años, la empresa Arrocera San Carlos ubicada en el este de la provincia de Chaco (Argentina) llevó a cabo un proceso innovador de diversificación productiva de gran envergadura. Tras un reclamo ambiental, en el año 2010 la empresa integró su actividad principal, el cultivo de arroz, con una actividad complementaria de base agroindustrial, el cultivo y procesamiento de pacú, bajo un esquema de rotación entre ambas producciones. De esta manera, se desataron cambios de magnitud en la organización que obligaron a coordinar una serie de actividades nuevas y complementarias al nuevo producto (procesamiento, logística y comercialización del nuevo producto, abastecimiento de insumos y requerimientos tecnológicos específicos). Lo que comenzó como respuesta a un reclamo comunitario sobre los efectos ambientales de la producción de arroz, (a) se transformó en un emprendimiento exitoso con fuertes implicancias económicas y tecnológicas para la empresa en cuestión, (b) diluyó las tensiones con la comunidad local y (c) abrió posibilidades para la entrada de nuevos emprendimientos de base piscícola en la región. El caso dio lugar a una serie de interrogantes en materia de desarrollo local relacionados a comprender las condiciones para la emergencia de pioneros en nuevas actividades productivas, y con éstos, el surgimiento de seguidores que consoliden la actividad en el espacio local.

En este contexto, el principal objetivo de este artículo es analizar las competencias organizacionales y productivas requeridas en un proceso de diversificación productiva al interior de una empresa, considerando en especial la proximidad de conocimiento entre la actividad principal de la firma y las actividades hacia las cuales se diversifica. Como objetivo secundario, desde una perspectiva de desarrollo local, resulta de interés evaluar las posibilidades de que otros actores dentro del sector de origen transiten procesos de diversificación similares al desarrollado por la empresa pionera.

Desde la perspectiva de las competencias organizacionales (Dosi y Marengo, 2000; Dosi y Teece, 1998), capacidades (Teece y Pisano, 1994; Teece et al., 1997; Winter, 2003) y rutinas de la firma (Nelson y Winter, 1982), la diversificación productiva requiere el desarrollo de importantes procesos de aprendizaje. En este caso, se los observa en la implementación de nuevas técnicas productivas para el cultivo del arroz, por un lado, y en la incorporación de la actividad ictícola y en el procesamiento y comercialización del pescado, por el otro. Tal como predice la literatura, el proceso requirió de esfuerzos endógenos a la firma como de articulación con actores externos que permitieran complementar capacidades internas y expandir los conocimientos 
de la organización.

Por otra parte, la literatura de pioneros sostiene que las primeras empresas en ingresar a una nueva actividad potencialmente allanan el camino a seguidoras al reducir costos y barreras de entrada (Hausmann y Rodrik, 2003). La empresa pionera es por lo tanto quien inicia un proceso de diversificación productiva hacia actividades aun no exploradas dentro del territorio ${ }^{2}$. La reducción de costos enfrentados por los seguidores derivada del surgimiento del pionero se deben a que las actividades de búsqueda y experimentación realizadas por la empresa pionera reducen la incertidumbre sobre condiciones productivas y tecnológicas de una nueva actividad en una región dada. La literatura señala que las externalidades tecnológicas o de conocimiento que genera un pionero sobre posibles seguidores pueden contribuir a la complejización del perfil productivo local y regional al abrir el camino hacia una nueva actividad o hacia nuevos mercados de exportación (Sabel et al., 2012).

El caso analizado permite reconsiderar algunas de las principales hipótesis de ambos conjuntos de literaturas: competencias organizacionales y pioneros; ya que pone de manifiesto la complejidad de los procesos de innovación y diversificación productiva, y sus implicancias tanto al interior de la organización, como en su relación con el entorno.

A través de un estudio de caso de tipo explicativo, en primer término, se busca identificar los factores claves que permitieron que este proceso de diversificación sucediera, haciendo especial hincapié en los aprendizajes cruzados entre las actividades emprendidas (Cantner y Hanusch, 2005) y las complementariedades productivas. En segundo término, se estudian las posibilidades de que surjan nuevos emprendimientos similares o incluso asociados a esta experiencia. En esta dirección, la pregunta de investigación es cómo la organización aprende en el proceso de incurrir en nuevas actividades productivas y cómo estos procesos trascienden a la organización con impactos de diferente tipo sobre el territorio.

Se entiende que la decisión de implementar la producción integrada de arroz y pacú disparó en la empresa un conjunto de decisiones de inversión que están dentro de un esquema de complementariedades productivas derivadas del propio proceso de diversificación (Breschi et al., 2003; Rumelt, 1982; Teece et al., 1994). De esta forma se estudian las diferentes áreas del modelo de negocios de la empresa y la forma en que se articulan entre sí. De tal modo, como innovaciones en cascada, la empresa tuvo que desarrollar nuevas rutinas y cambios organizacionales profundos en diversas áreas pero integrados entre sí. Por otra parte, se analiza el rol de las capacidades transversales a ambas actividades como condición necesaria para que el proceso de diversificación tuviera lugar. Por último también se considera el contexto de surgimiento de la innovación así como el impacto sobre posibles adoptantes en el futuro.

En esta dirección el caso permite reconsiderar las hipótesis de la teoría frente al fenómeno de la diversificación de una organización que incurre nuevas actividades

2 El caso analizado en este artículo, la Arrocera San Carlos constituye una empresa pionera según la definición ofrecida, en tanto es la primera empresa de la región (este de la Provincia de Chaco) en introducirse en el cultivo y procesamiento de pacú. 
productivas para ella y para el entorno local. Al mismo tiempo esta metodología abre el camino a la exploración de nuevas hipótesis basadas en la experiencia.

En la segunda sección se desarrolla el marco conceptual que integra la perspectiva evolucionista de la firma sobre diversificación productiva y la literatura de pioneros. En la tercera sección, se explica el diseño de investigación y la metodología utilizada. En la cuarta, se describe el caso analizado distinguiendo entre una primera etapa que actúa como el contexto en el que la innovación de la empresa tiene lugar y una segunda etapa en la que se complementa el proceso de diversificación productiva al surgir nuevas actividades en la firma, tanto en términos horizontales como verticales. En la quinta sección se discuten los resultados obtenidos.

\section{Diversificación productiva en firmas pioneras. El rol de las capacidades organizacionales y competencias}

La diversificación productiva es vista por diferentes autores como una estrategia positiva para el desarrollo económico. Esto se verifica tanto a nivel agregado, la diversificación de las exportaciones está asociada al crecimiento económico (Fagerberg, 2000; Hausmann y Hidalgo, 2010; Saviotti y Frenken, 2008), como a nivel regional o local, una región diversificada enfrenta una menor vulnerabilidad porque opera sobre diferentes mercados y porque amplía su base de conocimientos tecnológicos y productivos (Jacobs, 1961). No obstante, a nivel de firma, la evidencia así como los argumentos no son tan lineales. En este caso, las ventajas de la diversificación están asociadas a: (i) motivos de eficiencia debido a la existencia de activos indivisibles como ciertas capacidades o sinergias entre las diferentes actividades productivas, por lo que la diversificación le permite a la empresa aprovechar economías de alcance, (ii) costos de transacción, por lo que la firma elije internalizar alguna actividad antes que enfrentar los costos de uso de los mercados, (iii) la baja densidad de los mercados que no le permite a la empresa especializarse, o (iv) una estrategia de diversificación de riesgos derivada de operar en diferentes mercados (Breschi et al., 2003; Rumelt, 1982; Teece et al., 1994).

La literatura de la empresa basada en los recursos (Penrose, 1995; Peteraf, 1993; Wernerfelt, 1984) así como la perspectiva evolucionista de la firma (Cohendet y Llerena, 2003; Dosi y Marengo, 2000; Nelson, 1991; Nelson y Winter, 2002) analizan la cuestión de la diversificación de la empresa prestando especial atención a las capacidades de la firma y las formas de aprendizaje. La diversificación a nivel de firma implica que la organización, al emprender nuevos negocios, debe desarrollar nuevas capacidades específicas. La manera en que las organizaciones adquieren estas capacidades no es trivial, de modo que las posibilidades de cada firma de diversificarse hacia nuevas actividades están en función de su trayectoria previa y especialmente en función de las competencias organizacionales, comerciales, tecnológicas y productivas con las que cuenta la empresa y las que logra construir sobre la base de alianzas y vinculaciones con actores externos.

La teoría de la firma basada en los recursos como así también la teoría evolucionista reconoce que la heterogeneidad entre empresas se deriva de que cada organización cuenta con un acervo propio de recursos (activos, capacidades y rutinas) que organiza de una forma idiosincrática y combina con recursos externos. Dentro de 
esta perspectiva, la competitividad de una firma está basada en la existencia de recursos no triviales que la distinguen de la competencia. En este contexto, una empresa podrá diversificarse si uno de estos recursos no triviales puede usarse en más de un contexto de negocios. Si este activo refiere a la capacidad de usar una tecnología particular, es posible que la empresa expanda sus actividades hacia otras relacionadas con el uso de esta tecnología. Por otra parte, si el activo de la firma radica en el conocimiento de un mercado externo, en una marca posicionada en el mercado o en su capacidad de gestión, es posible que la firma expanda sus actividades hacia otras no relacionadas (Saviotti y Frenken, 2008).

Entrar en una nueva rama de actividad, no obstante, es una actividad innovadora para la empresa e implicará un cambio sustantivo en las rutinas y subrutinas de la organización. Según Nelson y Winter (1982) las firmas innovan cuando las rutinas existentes enfrentan problemas; luego, la innovación es vista como un cambio de rutinas derivado de problemas en las rutinas existentes. La revisión de rutinas puede ser producto de una búsqueda activa de la organización (rutinas de innovación) que persiguen la mejora continua o puede ser el resultado de un shock externo al que se enfrenta la empresa por cambios en su entorno. La empresa que innova, ya sea por uno u otro motivo, genera procesos de aprendizajes derivados primero de la búsqueda dentro de su repertorio de rutinas y segundo de la instauración de nuevas rutinas que incluyen adaptaciones de las existentes. Incluir un nuevo producto al mix de productos elaborados y ofrecidos por la empresa, implica cambios en las rutinas organizacionales de magnitud, más allá de que algunas de ellas se mantengan o se adecuen con pocos cambios a la nueva actividad. Por otra parte, la introducción de una nueva actividad puede ser oportunidad para introducir cambios en los procesos productivos de las actividades ya desarrolladas debido a complementariedades de conocimientos o productivas entre ambas.

No obstante, los aprendizajes internos a la organización no son los únicos que se derivan de procesos de innovación y de diversificación productiva. Hausmann y Rodrik (2003) y otros trabajos posteriores en la misma dirección (Mostafa y Klepper, 2017, Sabel et al, 2012, Artopoulos et al, 2013) han señalado el rol que juegan los pioneros como fuentes de externalidades y principales motores de la diversificación productiva a nivel local. De acuerdo con estos autores, las firmas que descubren nuevos mercados están sujetas a procesos de copia e imitación por parte de seguidores que se ahorran los costos de experimentación. Por lo tanto, los costos de aprendizaje que enfrentan los pioneros son más altos que los de los seguidores, permitiéndoles a estos últimos una mejor estimación de sus costos de producción antes de emprender una nueva actividad e ingresar a un nuevo mercado. Además de las externalidades de conocimiento, la presencia de una nueva actividad en el entorno local puede dar lugar a externalidades pecuniarias propias de las economías de aglomeración. Este tipo de externalidades refiere a la posibilidad de encontrar localmente insumos o servicios a menor costo que los de la competencia, derivados de la presencia de proveedores especializados en el entorno local. Es decir, posibles seguidores podrían hacer uso de servicios provistos por el pionero o por proveedores del pionero que ajustaron su oferta a la nueva actividad local. La escala de producción 
es en sí misma una externalidad pecuniaria que podría favorecer a la aparición de nuevos productores locales (David y Rosenbloom, 1990; Krugman, 1991; Perroux, 1950; Prebisch, 1959).

En el caso analizado se busca evaluar algunas de las hipótesis planteadas por ambos conjuntos de literatura y discutir la pertinencia de las mismas para la experiencia analizada. En primer lugar, las hipótesis de la literatura evolucionista sobre diversificación productiva a nivel de firma que argumentan que:

a.1. la diversificación productiva se apalanca sobre ciertos activos, capacidades y rutinas estratégicos de la firma que son comunes a ambas actividades (diversificación relacionada por capacidades) (Rumelt, 1982; Teece et al., 1997, 1994). Al mismo tiempo, los procesos de aprendizajes intra-organizacionales y los cambios en rutinas existentes, así como la incorporación de nuevas rutinas derivadas de los procesos de diversificación complejizan la base de conocimiento de la organización dentro de una coherencia organizacional (Teece et al., 1994);

a.2. la diversificación productiva, como actividad innovadora (introducción de un nuevo producto y proceso, además de los cambios organizacionales asociados) es una respuesta de la firma que se dispara como resolución de problemas en sus actuales rutinas (Nelson y Winter, 1982).

En segundo lugar, las hipótesis de la literatura de pioneros, referidas a que:

b.1. la integración productiva requiere que el emprendedor realice actividades de experimentación con el objetivo de reducir la incertidumbre que enfrenta sobre sus costos de producción y productividad (Artopoulos et al., 2013);

b.2. las barreras a la entrada de nuevos jugadores refieren a los costos de aprendizaje incurridos por el pionero, una vez que el pionero entra en el mercado los seguidores encuentran el camino allanado (Hausmann y Rodrik, 2003);

b.3. los costos de procesos de aprendizaje y construcción de capacidades y rutinas son abordados por la empresa de forma individual y por lo tanto si no se garantiza que se preserve el mercado de la competencia de otras empresas, habrá subinversión en el desarrollo de innovaciones que deriven en procesos de diversificación productiva (Hausmann y Rodrik, 2003; Sabel et al., 2012);

b.4. un nuevo productor en la región (en la localidad o inmediaciones) se enfrenta a menores costos al acceder a insumos críticos y servicios que se desarrollaron luego del ingreso del pionero o provistos por el mismo.

En ambos conjuntos de hipótesis están implícitos argumentos largamente explorados por la organización industrial que refieren a motores para el cambio, tales como: i) captura de externalidades (literatura de pioneros), ii) la búsqueda de complementariedad productiva entre actividades (literatura de capacidades organizacionales y diversificación productiva). En la sección de resultados, se exploran estos argumentos a la luz del caso estudiado, que permite reconsiderar algunos aspectos de las mismas. 


\section{Diseño metodológico}

Para llevar a cabo la presente investigación se utiliza un análisis de estudio de caso (Yin, 1994) a partir del cual se busca analizar el alcance de la teoría para dar cuenta de los procesos observados en este caso particular. El tipo de estudio de caso aplicado es explicativo. Pretende explicar los procesos derivados de una decisión de diversificación productiva que se desenvuelven en el tiempo y se interrelacionan con múltiples factores internos y externos a la organización. Una metodología cualitativa es lo más adecuado para abordar experiencias de este tipo en las que los/as investigadores/as no tienen control sobre los fenómenos que se desenvuelven en el tiempo. Por otra parte, estas metodologías permiten una aproximación flexible en la que se pueden explorar los límites de tales experiencias sin preconceptos definidos, y de esta forma considerar todas sus posibles implicancias. Los conceptos teóricos utilizados son puestos a prueba no con el objetivo de corroborarlos o refutarlos sino para construir hipótesis que conduzcan a una teoría basada en la evidencia (Glaser y Strauss, 1967).

Se realizan el estudio de caso sobre la empresa Arrocera San Carlos que luego del proceso de diversificación productiva conformó al grupo Puerto las Palmas, localizado en el este de la provincia de Chaco, Argentina, y el conjunto de actores de la comunidad que estuvieron involucrados en la evolución del caso, ya sea en el plateo de las demandas ambientales como aquellos que se vincularon (formal o informalmente) con las empresas del grupo para el desarrollo del proceso de diversificación productiva.

Se llevan a cabo entrevistas en profundidad con visita a dos de los establecimientos productivos. En total se realizan entrevistas a 4 personas de la empresa de entre 2 y 3 horas en cada caso, en una jornada de trabajo en la que se visitan los establecimientos de Arrocera San Carlos y Puerto las Palmas, que incluyó una vista a las piletas donde se siembra el pacú, la visita al frigorífico, las instalaciones donde se está construyendo un molino arrocero y un muelle sobre el río Paraguay. Las entrevistas fueron complementadas con un cuestionario por escrito en el que se solicitaron precisiones sobre cuestiones referidas a la propiedad de la tierra, formas societarias, estructura de costos de producción bajo diferentes modelos de producción (uso de agroquímicos, energía, trabajo, etc.), orígenes del financiamiento y vinculaciones con organismos públicos y privados, entre otras cuestiones. También se realizan entrevistas a actores del gobierno provincial, así como a exponentes de la comunidad. En estos casos, fueron 2 entrevistas de entre 1 y 2 horas. una oportunidad para promover el desarrollo sustentable en las comunidades locales.

Tabla 1. Entrevistas realizadas

\begin{tabular}{|l|l|l|l|}
\hline $\begin{array}{l}\text { Nombre del/de la } \\
\text { entrevistado/a }\end{array}$ & Rol & $\begin{array}{l}\text { Lugar de la } \\
\text { entrevista }\end{array}$ & Duración \\
\hline Eduardo Meichtry & Empresario fundador. & $\begin{array}{l}\text { Establecimientos } \\
\text { productivos. }\end{array}$ & 4hs \\
\hline
\end{tabular}




\begin{tabular}{|c|c|c|c|}
\hline Martín Meichtry & $\begin{array}{l}\text { Hijo del empresario } \\
\text { fundador. Agrónomo } \\
\text { especializado en } \\
\text { rotación de arroz y } \\
\text { pacú. }\end{array}$ & $\begin{array}{l}\text { Establecimientos } \\
\text { productivos. }\end{array}$ & $4 \mathrm{hs}$ \\
\hline $\begin{array}{l}\text { María Eugenia } \\
\text { Meichtry }\end{array}$ & $\begin{array}{l}\text { Hija del empresario } \\
\text { fundador. Contadora } \\
\text { de la empresa. }\end{array}$ & $\begin{array}{l}\text { Resistencia, } \\
\text { Chaco. }\end{array}$ & $2 \mathrm{hs}$ \\
\hline Nestor Gromenida & $\begin{array}{l}\text { Consultor en } \\
\text { piscicultura. }\end{array}$ & $\begin{array}{l}\text { Establecimientos } \\
\text { productivos. }\end{array}$ & 4 hs \\
\hline Enrique Orban & $\begin{array}{l}\text { Ministro de } \\
\text { Producción y } \\
\text { Ambiente en el } \\
\text { momento de la } \\
\text { implementación de la } \\
\text { rotación y actual } \\
\text { director del centro } \\
\text { regional Chaco- } \\
\text { Formosa de } \\
\text { Servicio Nacional de } \\
\text { Sanidad y Calidad } \\
\text { Agroalimentaria } \\
\text { (SENASA). }\end{array}$ & Resistencia, Chaco & $2 \mathrm{hs}$ \\
\hline Rolando Núñez & $\begin{array}{l}\text { Presidente de la } \\
\text { Fundación Mandela, } \\
\text { principal } \\
\text { impulsor de las } \\
\text { demandas por } \\
\text { cuestiones } \\
\text { ambientales. }\end{array}$ & Resistencia, Chaco & $2 \mathrm{hs}$ \\
\hline
\end{tabular}

Fuente: elaboración propia en base a entrevistas.

En forma adicional se recolectó información secundaria de diarios locales y nacionales (Diario Norte, Chaco día por día, Página/12, La Nación), revistas especializadas (suplementos Norte Rural, La Nación Rural), páginas web, informes de centros de investigación (Instituto Nacional de Tecnología Agropecuaria, INTA) y documentos oficiales referidos al conflicto ambiental (expedientes e informes técnicos). Por otra parte, se accedió a las tesis de grado y otras publicaciones en eventos científicos presentadas por los hijos de Meichtry.

\section{Desarrollo del caso}

\subsection{Antecedentes}

Eduardo Meichtry proviene de una familia de arroceros y es Ingeniero Agrónomo formado en la provincia de Entre Ríos. A finales de los setenta se radica en Chaco 
para dedicarse a la producción de arroz. Desde entonces y por más de 35 años su principal producción fue arroz cáscara, un producto de bajo valor agregado, que comercializaba al Grupo Estrella, entonces dueño de la marca de arroz Gallo. En el año $2008^{3}$ la empresa fue sujeto de una serie cuestionamientos por el uso creciente de agroquímicos en su producción, impulsados por diversos actores de la comunidad local y grupos ambientalistas de alcance regional y nacional.

La empresa enfrentó demandas judiciales con medidas concretas como restricciones a la fumigación (en una medida interpuesta por vecinos de La Leonesa y Las Palmas junto a productores, solicitan amparo "ambiental" y medida cautelar innovativa) y con solicitudes de informes técnicos de impacto ambiental de sus actividades.

A pesar de que los resultados de los informes ambientales fueron favorables para la empresa no cesaron los reclamos comunitarios, que sostenía que las mediciones de contaminación eran falaces porque las solicitaba la misma empresa ${ }^{4}$. La movilización comunitaria fue recogida por medios de prensa que le daba visibilidad pública y escaló a la prensa nacional ${ }^{5}$. Esto atrajo a exponentes de movimientos ambientalistas y demás organizaciones de nivel nacional que venían alertando sobre el uso de herbicidas y en especial del glifosato desde hacía tiempo (entre ellos el Doctor Carrasco, embriólogo, investigador del Consejo Nacional de Investigaciones Científicas y Técnicas, CONICET).

En el marco de este conflicto, se da la incorporación de los hijos del empresario a la empresa. El conflicto ambiental penetra en las relaciones familiares y se manifiesta en una crisis de continuidad de la actividad tal como venía realizándose.

El reclamo social alcanzó tal grado de complicación que obligó a la familia a repensar una estrategia que permita renovar la imagen de la empresa más allá de los resultados de los estudios ambientales y de la resolución judicial del conflicto, que todavía se encontraba pendiente. En sus propias palabras urgía demostrar ante la población que la producción responsable de arroz no genera riesgo de contaminación de ambiente (suelo, agua, flora-fauna) ${ }^{6}$.

Hacia 2010, la empresa se propuso demostrar la ausencia de contaminación a partir de la creación de un estanque de 18 ha con peces, en las parcelas lindantes con la zona urbana, bajo la asunción de que los peces son un indicador sensible de contaminación (Ibarra, 2005). A esta actividad piloto, la empresa la complementó con una actitud activa y abierta hacia la comunidad, publicando los resultados de las evaluaciones ambientales, promoviendo actividades locales y generando una imagen de marca de la compañía con la que la comunidad se identificara.

Para desarrollar el caso testigo de los peces, la empresa requería de conocimientos

3 En el contexto de reclamos por tierras y de una creciente demanda social por cuestiones ambientales en la provincia de Chaco y otras regiones del país.

4 Esto surge tanto de la entrevista con Rolando Núñez de la Fundación Mandela, como del fallo de segunda instancia de la Cámara de Apelaciones en el que ordena al juez volver a las medidas iniciales justificado en el hecho de que las pruebas de no contaminación eran ofrecidas por la propia empresa (Diario Norte, 19/03/2011).

5 Diario Página/12, 14/06/2010, 17/08/2010 y 18/03/2011. Diario La Nación, 11/12/2010.

6 Entrevista a Eduardo Meichtry. 
específicos sobre piscicultura para lo que se contactó al Ingeniero Agrónomo Néstor Gromenida, como consultor externo, quien venía trabajando en esta actividad en Clorinda (provincia de Formosa) pero a menor escala ${ }^{7}$. En un primer momento los peces fueron sembrados sin un fin comercial, pero el Ingeniero Gromenida mostró la potencialidad del negocio de combinar la producción de arroz con la piscicultura sobre la base de un esquema de rotación y siembra de arroz pregerminado que a su vez redujera el uso de agroquímicos.

El emprendimiento despertó el interés de diversos funcionarios públicos e investigadores, dado el potencial para el desarrollo productivo de la región y la creación de empleo. La propuesta resultó interesante porque sería potencialmente aplicable en amplias extensiones de tierra de la provincia debido a la "gran cantidad de hectáreas con las mismas características de suelo" (categoría IV/V, no apto para soja o maíz) ${ }^{8}$.

Cabe destacar que el vínculo con el piscicultor formoseño no solo fue funcional para hacer frente a la cuestión del conflicto ambiental, sino que también impulsó la idea innovadora de la rotación entre pacú y arroz como una propuesta productiva válida. Esta nueva actividad trajo desafíos nuevos en materia de procesamiento, comercialización y logística que obligaron a la empresa a entrar en nuevos negocios. El proyecto de rotación escaló de las 18 ha destinadas al caso testigo a 900 ha que actualmente se destinan a la rotación.

\subsection{Rotación arroz-pacú: capacidades complementarias}

Néstor Gromenida fue funcionario del gobierno de Formosa y emprendedor piscícola en esa provincia. Había recorrido varios países estudiando experiencias de producción ictícola ${ }^{9}$. Gromenida, en forma personal lleva adelante un emprendimiento de pacú en pequeña escala que desarrolla junto a su familia. Sin embargo, los problemas de costos asociados a la pequeña escala y al procesamiento artesanal derivaron en una baja productividad que atentaba contra la rentabilidad de la actividad. A pesar del fracaso económico, este emprendimiento resultó clave para el desarrollo de los conocimientos específicos en pacú, no solo sobre el ciclo de crecimiento, alimentación y reproducción, sino también sobre el tamaño óptimo para fines gastronómicos, en materia de procesamiento, despinado completo e identificación de diferentes cortes y productos posibles. La experiencia de más de 25 años en piscicultura tanto en desarrollos de esquemas de explotación, como en su propio emprendimiento, lo dotaban de un conocimiento profundo en la actividad, aunque sin experiencia concreta en explotaciones a gran escala.

El conocimiento aportado por el consultor Gromenida, le permitió a la arrocera

7 Establecimiento Isla pé Acuicultura, que a partir del 2002, comienza a desarrollar productos especiales a base de pacú para los restaurantes de la provincia y el gusto de los consumidores de la zona.

8 Según informe del INTA (2000), Clase IV: los suelos tienen muy severas limitaciones que reducen la elección de cultivos, o que requieren un manejo muy cuidadoso, o ambos. Preferiblemente para ganadería. Clase V: los suelos no tienen riesgo de erosión, pero tienen otras limitaciones, como exceso de humedad, poco prácticas de eliminar, que limitan su uso a ganadería.

9 Diario La Nación, 01/08/1998. 
chaqueña implementar el esquema de rotación que significó la apertura a una nueva gama de actividades productivas no solo primarias, sino agroindustriales. Asimismo, la rotación le permitió utilizar nuevas técnicas con menor uso de agroquímicos, origen del conflicto que los incentivó a adentrarse en esta nueva actividad. La rotación arrozpacú torna a la producción de arroz mucho más sustentable en cuanto al aporte de fertilizantes, herbicidas o labranza. La siembra de pacú sobre barbecho, dada su característica omnívora, posibilita que los peces se alimenten de restos de arroz y plagas, en particular del caracol que ataca al cultivo de arroz en su primera etapa. Por otra parte, al drenar los estanques luego del ciclo de pacú es posible sembrar arroz pregerminado sobre el barro saturado, libre de caracoles y malezas, e inundar luego. Este proceso permite minimizar el uso de herbicidas. Por otra parte, las heces del pacú actúan como fertilizante natural.

La rotación permite una mayor eficiencia en comparación con el monocultivo ya sea de arroz o pacú, en aspectos económicos, ambientales, uso de energía y agua. Se genera una sinergia entre ambas actividades: la rotación permite por un lado, realizar el cultivo de arroz con un laboreo mínimo y mayor sustentabilidad. Por otro lado, el aprovechamiento de la infraestructura de drenaje para el vaciado sanitario requerido en la producción de pacú, evita que se desarrollen patologías en los estanques, que sin este sistema quedaría ocioso hasta la próxima campaña ${ }^{10}$.

Además de las complementariedades tecnológicas mencionadas, las producciones comparten saberes tácitos y rutinas productivas que favorecen la implementación de esta nueva actividad en productores de arroz. Entre ellas, las rutinas referidas a la gestión de aguas, algo que no se derivaba de la experiencia de Gromenida en pequeña escala pero sí de la producción arrocera realizada por Meichtry. Mientras que los conocimientos sobre el pacú, su comportamiento en corrientes de agua (que facilitan la cosecha ${ }^{11}$ ), su ciclo de engorde y reproducción, requirió de desarrollo de nuevas rutinas que eran adaptaciones de las que aplicaba Gromenida en pequeña escala.

\subsection{Resultados de la innovación: impulso a la integración vertical y horizontal}

El desarrollo de la piscicultura impulsó un proceso de integración vertical tanto "aguas arriba" (insumos) como "aguas abajo" (elaboración y comercialización) con crecientes complementariedades productivas. Dada la escala del proyecto, se aprovecharon las complementariedades de los diversos eslabones de la cadena productiva del pacú. Sin embargo, no fueron los mismos los factores que incentivaron la integración de cada uno de estos.

10 Los detalles sobre las mejoras de costos derivadas del esquema de rotación de arroz y pacú, las complementariedades entre ambas actividades, impactos ambientales y el lapso temporal en el que el modelo de negocio se torna consistente considerando el repago de inversiones se pueden encontrar en la tesis de graduación de Marcos Meichtry de 2014 titulada "Balance hídrico y energético de la rotación arroz-pacu en terminos de rentabilidad potencial por su eventual sinergia", y en bibliografía complementaria como (Meichtry, 2015).

11 El pacú nada contra la corriente, lo que condujo a innovaciones incrementales que favorecen la cosecha al atraer a los peces por las corrientes generadas artificialmente. Entrevista realizada a Néstor Gromenida. 
En primer término cabe mencionar cuáles fueron las nuevas actividades desarrolladas por la firma a partir de la integración y cuáles se derivan de planes previos de expansión. También conviene distinguir entre las actividades planeadas y las efectivamente emprendidas (ver en Gráfico 1 los cuadros con fondo gris y los cuadros con fondo blanco).

La introducción de la producción de pacú, demandó la implementación de un esquema de cosecha, procesamiento y comercialización. En primer lugar, uno de los problemas habituales de la piscicultura en pequeña escala es la imposibilidad de ofrecer un flujo constante de producto por el ciclo de engorde, y la necesidad de drenar el estanque para evitar la aparición de enfermedades. La experiencia relatada se desarrolla bajo un esquema de producción de pacú semi-extensivo. La baja densidad de peces por metro cuadrado minimiza los riesgos de enfermedades sin tener que aplicar productos de sanidad animal. No obstante, requiere alimentación suplementaria, en particular en la etapa de engorde.

En el caso estudiado, los productores desarrollaron circuitos de engorde que permiten ir capturando animales de tamaño óptimo para su procesamiento y comercialización a lo largo de todo el año. Este elemento los distingue de la competencia de pequeña escala intensiva ya que llegan a la demanda de modo continuo (lo que es especialmente valorado en restaurantes ${ }^{12}$ ). En segundo lugar, el pacú para ser comercializado debe ser procesado en una planta industrial. La empresa cuenta con su propio frigorífico que cumple con los requisitos regulatorios (SENASA) en el que se procesa la totalidad de la producción y opera con excedente de capacidad instalada. En la planta trabaja personal capacitado en la limpieza de los animales, el despinado (que surge de un estudio del patrón de espinas del pacú desarrollado por Gromenida), el procesamiento en productos elaborados, y en el envasado y congelado. Esta unidad productiva resultó fundamental para el procesamiento de pescado en gran escala.

Una actividad contemplada adicionalmente es brindar servicios de procesamiento a productores piscícolas de menor tamaño relativo. La industrialización (frigorífico), comercialización y marketing requiere disponer de un volumen de producción adecuado que sería difícil que un pequeño productor lo lograra por sí mismo. Algunas apreciaciones indican que "la importancia de un emprendimiento grande, como el encarado por el empresario arrocero es lo que traccionará la producción de los más chicos que podrán integrarse sumando su producción para aprovechar los procesos posteriores en las esferas de industrialización y comercialización asegurando el éxito de la nueva actividad" (Diario Norte, 09/02/2011).

Del procesamiento del pacú obtienen dos subproductos, harina y aceites de pescado, que se utilizan en la producción de alimentos balanceados. Estos subproductos provienen del procesamiento que ellos mismos realizan de los desperdicios de la faena (cabeza y espinazo), en una grasería de la zona pero de propiedad de terceros, que están considerando remplazar por una propia que opere en escala eficiente.

12 Entrevista a Martín Meichtry. 


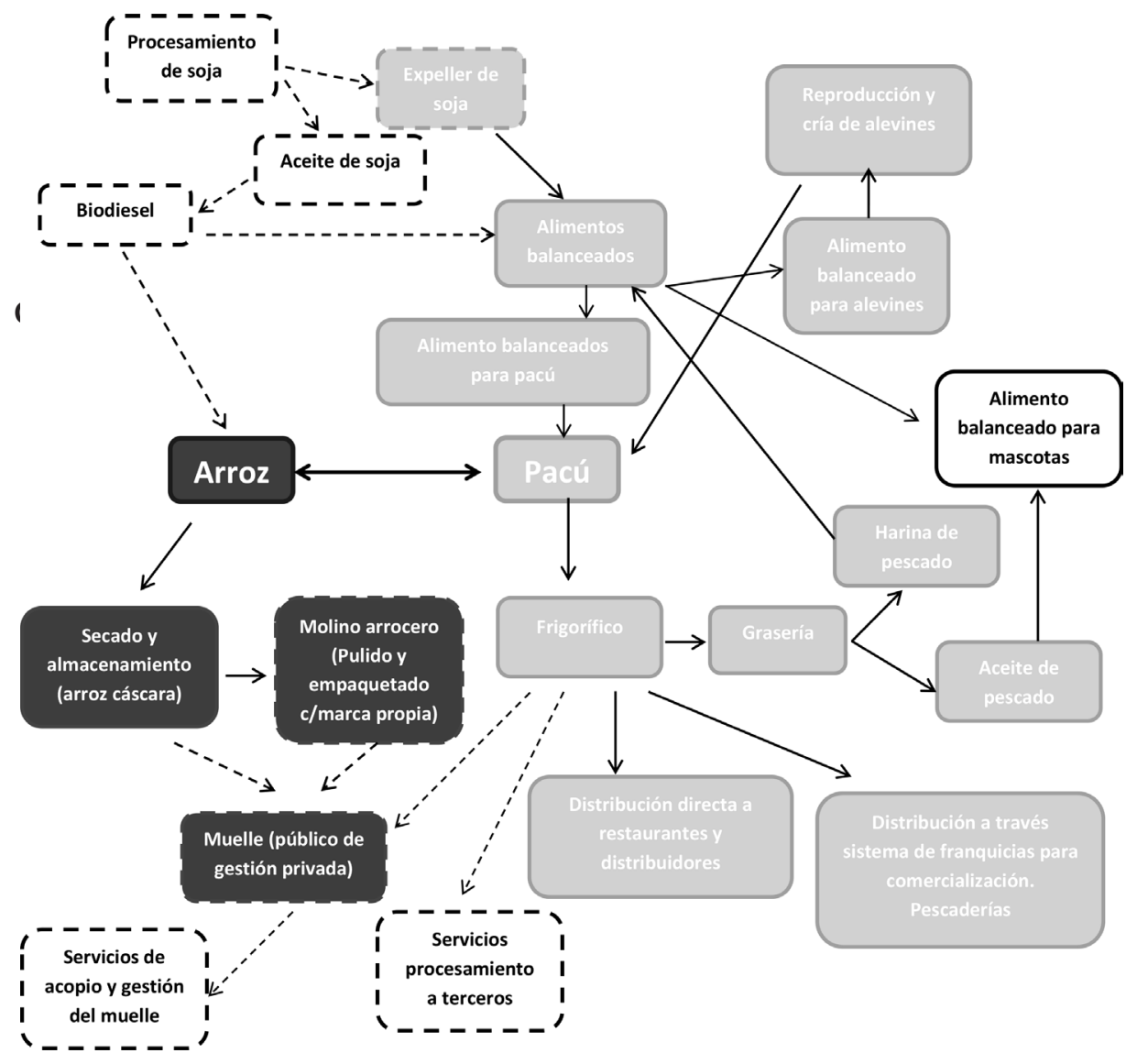

\section{Notas:}

Línea punteada: actividades y productos planeados (en diferentes etapas de ejecución).

Línea sólida: actividades y productos realizados por la empresa.

Fondo gris con letra blanca: diversificación relacionada al Pacú.

Fondo negro con letra blanca: diversificación relacionada al arroz.

Fondo blanco con letra negra: diversificación no relacionada.

Fuente: elaboración propia sobre base de entrevistas y fuentes documentales consultadas. 
En el caso del alimento balanceado, dado que este insumo representaba el $70 \%$ del costo de producción de pacú, se generaba una fuerte dependencia respecto de agentes externos no especializados (se compraba el alimento a productores). La volatilidad del precio y calidad ponían en riesgo la rentabilidad del nuevo producto ${ }^{13}$. Por otro lado, existían incentivos vinculados al aumento de la eficiencia. La producción de alimento balanceado permitía el aprovechamiento de las partes del pacú que en principio se desperdiciaban (cabeza, espinazo, cola y cuero).

Este proceso de integración vertical a su vez impulsó una mayor diversificación productiva horizontal. Con el objeto de mejorar la eficiencia en el uso de la capacidad instalada de la planta de alimentos para pacú, y utilizar el aceite de pescado que anteriormente se desechaba, surgió la idea de producir alimento balanceado para perros. Por su parte, la harina se comenzó a utilizar como complemento proteico del alimento balanceado producido para su propia cría de alevines (requiere más proteína animal que los peces más grandes).

La producción del alimento balanceado hoy requiere de la compra de expeller de soja. No obstante, en los planes de la compañía está procesar el grano de soja obteniendo el expeller para la elaboración de los alimentos balanceados y transformar en biodiesel el aceite derivado del prensado de la soja. Calculan que el biocombustible generado por esa vía alcanzaría para garantizar el autoabastecimiento de energía dada la escala de producción.

Para la comercialización, la empresa tuvo que realizar un esfuerzo importante de desarrollo y posicionamiento de la marca propia para los productos derivados de pacú. En forma adicional, estableció canales específicos de comercialización no abiertos con anterioridad. El consumo de pacú y otros pescados de río en la localidad y en las zonas aledañas estaba vinculado a la pesca artesanal y a actividades recreativas, valorado pero ocasional. En este contexto, la empresa implementa una búsqueda por expandir el mercado local y regional de pacú llegando a las familias, tratando de fomentar un consumo cotidiano. Para ello, la empresa establece una red de franquicias con las cuales llega a diferentes barrios y localidades de la provincia y provincias aledañas, tratando de que su marca sea identificada con un producto de calidad "listo para preparar y llevar a la mesa". Esto requirió de importantes esfuerzos de aprendizaje que ya habían sido emprendidos por el ingeniero Gromenida en su experiencia previa en Formosa. Gran parte de los productos ofrecidos por la empresa ya habían tenido una experimentación en el mercado formoseño, aunque en menor escala. Entre ellos, los conocimientos claves del mercado fueron: (i) ofrecer un producto sin espinas, siendo que el pacú se caracterizaba en el saber popular por ser un pescado de muchas espinas, (ii) ofrecer un producto congelado en porciones pequeñas, (iii) identificar el tamaño óptimo para su presentación en el plato, y (iv) optimizar el tamaño de faena y llegar al mercado con un producto magro, entre otras. Este aprendizaje, al mismo tiempo, les permitió descartar otras opciones, por ejemplo, las hamburguesas de pacú que quisieron ser introducidas en cadenas de comida rápida sin éxito, lo que enfatizó la relevancia de llegar con estos productos a las familias para su consumo cotidiano.

13 Actualmente solo se compra expeller de soja, maíz, trigo y el núcleo vitamínico-mineral. 
Por otra parte, el nombre de la marca y su slogan (Teko, pacú arrocero) ponen de relieve que el método de producción es sustentable y seguro, lo que les permite por un lado, hacer el producto atractivo a un público diverso, y por el otro, mejorar la imagen de la empresa en la comunidad. El trabajo de posicionamiento de la marca incluyó otras acciones: participar del programa Pescado Para Todos (un programa social de la provincia destinado a ofrecer productos a costo de productores), promover actividades recreativas y culturales como las fiestas internacionales de la pesca de pacú arrocero iniciadas en el 2014, además de dar charlas informativas y cursos de cocina.

La comercialización a través de la red de franquicias les dio visibilidad en diferentes puntos, ya que estas ofrecen exclusivamente productos de la empresa y exhiben la marca. En forma adicional, comercializan sus productos a través de la cadena regional de carnicerías Friar (Vicentin) y actualmente se busca acceder a otras cadenas de alcance nacional. La participación en estas cadenas amplía los puntos de venta y les permite llegar a localizaciones más lejanas del centro de producción. Por último, la empresa vende a distribuidores con llegada a restaurantes y hoteles en diferentes puntos del país. Esta estrategia de tres canales principales en su red de distribución (franquicias, cadenas regionales y nacionales, y distribuidores) les permite mantener un flujo constante de producción e ingresos mientras procuran ampliar la llegada al mercado familiar con un producto de precio competitivo, y acceder a mercados internacionales donde el consumo de pescado es mayor (Paraguay y Bolivia).

\subsection{Subunidades de negocio derivadas del arroz. Nuevas complementariedades}

De forma concomitante a este proceso, la empresa tenía un plan de expansión de actividades en arroz, que fue desarrollándose a la par de la implementación de la integración productiva (ver Gráfico 1 los cuadros con fondo negro y los cuadros con fondo blanco). Estos planes pudieron comenzar a implementarse únicamente luego de revertir la mala imagen de la empresa derivada del conflicto ambiental.

Este plan incluía, en primer lugar, construir su propio molino arrocero (actualmente con capacidad de producción de arroz integral y próximo a incorporar la etapa de pulido), financiado principalmente con un crédito del Banco de la Nación Argentina. Con el molino la empresa busca fundamentalmente brindar servicios a otros arroceros de la zona y eventualmente llegar al mercado con un arroz diferenciado de marca propia. La búsqueda de la diferenciación en arroces se complementa con esfuerzos tecnológicos realizados en colaboración con INTA para el desarrollo de nuevas variedades. En segundo lugar, realizar gestiones para la construcción de un muelle en terrenos propios sobre el río Paraguay que permita la salida de la producción de arroz directamente a diversos mercados internacionales. En la actualidad la construcción del muelle se está llevando a cabo con financiamiento público y promete mitigar los problemas de competitividad de la región referidos a los costos de transporte.

\section{Discusión de resultados y reflexiones}

\subsection{Respecto de las hipótesis de la literatura evolucionista}


Los resultados relatados hasta aquí argumentan a favor de las hipótesis planeadas por la literatura evolucionista y basada en los recursos de la firma, presentados en la sección 2.

La primera hipótesis se verifica en el caso estudiado al detectarse capacidades complementarias entre la empresa estudiada y el consultor experto en piscicultura. En forma adicional, la implementación de la rotación genera aprendizajes propios de la experiencia que no estaban disponibles en los repertorios de las rutinas de ninguno de los dos actores. En primer lugar, las capacidades de Meichtry refieren a la gestión de aguas y suelos que le permiten adecuar los terrenos de producción de arroz al desarrollo de la piscicultura en escala semi-extensiva. En segundo lugar, los conocimientos sobre la producción de arroz le permiten identificar con facilidad complementariedades productivas con la piscicultura, por ejemplo, en los requerimientos nutricionales del suelo y control de plagas, que se ven favorecidos por la presencia de la actividad ictícola (alimentación parcial de pacú a base de caracoles, y malezas, y fertilización natural del suelo derivadas de la presencia del pacú). Algunas innovaciones complementarias derivadas de la integración productiva refieren al uso de las aguas de las piletas de pacú para riego, aprovechando de esta forma los aportes nutricionales de las mismas al cultivo de arroz. Además, Meichtry cuenta con recursos complementarios entre ambas actividades referidas a aquellos activos fijos necesarios para implementar piscicultura a gran escala (tierras con acceso a agua, canales de riego, electrificación, etc.) y maquinarias apropiadas para la gestión de suelos y aguas (bombas, maquinaria agrícola y maquinaria específica para el acondicionamiento de terrenos).

Por otra parte, Gromenida ofrece conocimientos sobre la producción piscícola en pequeña escala y sobre la comercialización del pacú (aspectos de logística y desarrollo de mercado). Sus conocimientos productivos, construidos a partir de su experiencia en Formosa, son de dos tipos. En primer lugar, los genéricos, aplicables a diferentes modalidades y escalas de producción, se refieren fundamentalmente al ciclo del pacú (reproducción, cría, recría y engorde), necesidades de alimentación y aspectos biológicos del proceso. En segundo lugar, aporta conocimientos altamente específicos sobre procesos productivos de pequeña escala (entre 8 ha y 20 ha) y sin rotación, que constituye su principal experiencia. Estos conocimientos requirieron de adecuación y desarrollo a las nuevas condiciones, que se logaron a través de la práctica. La producción en mayor escala y bajo el esquema de rotación les permite operar a baja densidad, optimizando los procesos de alimentación natural (arroz y rastrojos) y suplementaria a las nuevas condiciones y, en segundo lugar, pueden experimentar con nuevas técnicas de cosecha a tanque lleno para mantener el flujo constante de faena. Los conocimientos sobre el mercado del pacú y las formas de comercialización, también son aportes clave de Gromenida al desarrollo de la actividad (cortes, desespinados, productos preparados, flujo continuo de faena, etc.), mientras que la perspectiva de los hijos del empresario aportan la visión de generar marca propia y la estrategia de comercialización a través de tres canales. Es interesante notar que las interacciones entre la empresa y el consultor especializado aportan oportunidades de aprendizaje y construcción de nuevas capacidades en 
ambos actores.

En cuanto a la segunda hipótesis, referida a la innovación como respuesta ante fallos en las rutinas actuales, efectivamente se observa en el caso presentado que la diversificación productiva como actividad innovadora es una respuesta de la firma que se dispara ante problemas en sus actuales rutinas. El caso relatado muestra que la integración productiva no se dispara de la búsqueda de las cuasi-rentas sino de la resolución de un problema comunitario originado en el reclamo ambiental. Una vez puesto en producción revela su conveniencia tanto como estrategia para mejorar la imagen de la empresa en la comunidad como para mejorar la eficiencia productiva de los establecimientos productivos al aplicar la producción conjunta de arroz y pacú. En lo que respecta a la resolución del conflicto ambiental, se percibe una disminución de la efervescencia del conflicto, con una comunidad más proclive a reconocer los aportes de la empresa a la vida cotidiana. La empresa por su parte, adoptó una nueva política comunicacional volviéndola más transparente ante los ojos de la comunidad. La colocación de la marca del pacú arrocero y de otros productos de la empresa, como los alimentos para mascotas, en la localidad donde se origina el conflicto y localidades de la región, favoreció la reducción de la conflictividad. Por otra parte, también realizaron actividades de promoción ya sea orientadas a la comunidad (concurso de pesca, fiesta del pacú arrocero) o a actores externos que validan la experiencia (asociaciones de productores, Aacrea (Asociación Argentina de Consorcios Regionales de Experimentación Agrícola), Aapresid (Asociación Argentina de Productores en Siembra Directa), Acsoja (Asociación de la Cadena de la Soja Argentina), instituciones de ciencia y tecnología, INTA, sector público, provincial y nacional).

Por otra parte, la integración productiva derivó en beneficios económicos para la empresa, ya que la producción conjunta permitió incrementar el valor agregado por hectárea en las parcelas en las que se practica la rotación. También se verifican beneficios adicionales, como riego con agua enriquecida, minimización de los tiempos ociosos de las tierras, diversificación de riesgos comerciales y nuevas capacidades productivas hacia áreas industriales, todos ellos elementos sin dudas positivos, aunque difíciles de determinar con precisión su contribución monetaria a los beneficios de la empresa.

En síntesis, el desarrollo de actividades desencadenadas a partir de la implementación de la integración productiva en el caso estudiado denota que la experimentación del emprendedor resulta clave en un contexto de incertidumbre sobre sus costos de producción y productividad. Este elemento, en última instancia, es el que justifica la protección de las cuasi-rentas del pionero obtenidas por ingresar primero en el mercado.

\subsection{Respecto de las hipótesis de la literatura de pioneros}

En cuanto al segundo grupo de hipótesis basado en la literatura de pioneros se observan las siguientes cuestiones.

En relación a la primera hipótesis, sobre incertidumbre asociada al proceso innovativo, el caso muestra que la incertidumbre del productor es total al comienzo de la actividad (piscicultura). Al punto que ni siquiera la planea en un comienzo 
como una actividad productiva rentable adicional al cultivo del arroz. En la reducción de esta incertidumbre la propia experimentación juega sin dudas un papel crucial, lo que queda demostrado en la ampliación paulatina de la escala de producción y superficie cultivada con pacú. No obstante, también cabe destacar, el rol de la red de contactos sociales, partners tecnológicos y políticas públicas en la reducción de estas incertidumbres. El mismo desarrollo de la actividad permitía mejorar el conocimiento del negocio reduciendo las incertidumbres sobre los aspectos tecnológicos, productivos y de mercado. Respecto a los conocimientos tecnológicos y productivos, la vinculación con Gromenida fue fundamental, no obstante, su propia experiencia requería ser adaptada a la producción en mayor escala, por lo que la incertidumbre se reducía, pero sin eliminarse por completo. Gromenida tampoco podría hacer una estimación cabal de los riesgos tecnológicos y productivos, en tanto no tenía experiencia directa en la rotación y las potencialidades en materia de complementación productiva estaban por ser reveladas. El conocimiento de mercado del nuevo producto, también lo proveía Gromenida, aunque no en la escala de producción esperada de este nuevo emprendimiento. Se reconocía la importancia de las redes de distribución por varios canales y la adecuación del producto al gusto del público. Sin embargo, quedaba por testearse la posibilidad de expansión de los mercados para el pacú (especialmente el familiar), algo que no había sido explorado hasta la fecha por el bajo desarrollo de la piscicultura a nivel nacional. En este aspecto, el diseño de la estrategia de posicionamiento de una marca en el mercado, que contó con un fuerte impulso de Martín Meichtry.

Con respecto a la segunda hipótesis, referida a barreras a la entrada sobre los seguidores, el caso de la empresa familiar analizada como pionera requiere de un estudio más minucioso de las posibles externalidades generadas sobre otros productores piscícolas o arroceros. A la fecha si bien la experiencia ha sido presentada en la comunidad en diferentes ocasiones como un caso de éxito, todavía no se observan seguidores. El estudio realizado muestra que las barreas a la entrada de nuevos jugadores no solo refieren a capacidades tecnológicas o información de mercados, sino también a complementariedades productivas que actúan como potentes economías de escala y alcance (ver hipótesis b.4, referida a los menores costos enfrentados por los seguidores.).

Sin embargo, los resultados muestran que muchas actividades de experimentación que realizó la empresa podrían evitarse en nuevos productores. Hoy existen estudios que analizan comparativamente el desarrollo de cada una de las actividades por separado y también en forma conjunta. Tanto el conocimiento tecnológico, como las habilidades laborales y el conocimiento de mercado, son saberes que se van radicando en la localidad y trascienden a la empresa analizada. Entre los saberes tecnológicos, encontramos al conocimiento sobre el ciclo biológico del pacú, las necesidades nutricionales en cada etapa, la densidad adecuada, las formas de pesca y de faena, entre otras. Estos conocimientos son difundidos por la empresa en diferentes tipos de eventos y redes de productores. Por otra parte, en lo que respecta a las habilidades laborales la empresa incursionó en múltiples actividades de formación (para las que contó con apoyo del Ministerio de Trabajo y Seguridad 
Social de la Nación). Las nuevas empresas que entren en la actividad posiblemente encontrarán mano de obra calificada en actividades tales como cosecha de pacú, fileteado y otras competencias complementarias como higiene y seguridad en una planta frigorífica más otras habilidades ya disponibles en la zona como manejo de aguas y utilización de maquinaria para manejo de suelos. Por último, en lo que respecta a los conocimientos de mercado, se observa que nuevos entrantes dispondrán de una demanda local más permeable al consumo de pescado y una demanda nacional creciente.

En cuanto a la tercera hipótesis, del caso presentado puede extraerse como lección que el éxito de la experiencia no depende exclusivamente de esfuerzos endógenos que la firma realiza en búsqueda de cuasi-rentas, sino que es el resultado de un proceso que está liderado por la empresa, pero que tracciona capacidades y aprendizaje de otras organizaciones, instituciones públicas y privadas. Por ejemplo, en el caso analizado participaron: i) consultores externos, en especial en lo que refiere a la adquisición del conocimiento tecnológico sobre piscicultura, con el aporte de expertos locales e internacionales, ii) distintas regionales del INTA, (en un trabajo conjunto con el programa de arroz de INTA desarrollado en Concepción del Uruguay), iii) SENASA, para la planificación de las plantas industriales tanto del frigorífico, verificando el cumplimiento de las normas técnicas asociadas al procesamiento de pescado, como de la planta de alimentos balanceados y sus posibles ampliaciones. En forma adicional a los aportes de conocimiento, desde la política pública hubo aportes y apoyos concreto a la expansión de la actividad en la región a partir de la construcción de infraestructura pública. Un ejemplo de estos aportes es la contribución del Programa de Servicios Agrícolas Provinciales (PROSAP), tanto en la financiación del muelle como en el tendido eléctrico rural sobre el río Bermejo. Por otra parte, desde la provincia se favoreció el desarrollo de la actividad a partir del apoyo brindado por el Ministro de Producción.

Finalmente, en cuanto a la cuarta hipótesis se observa que luego de la diversificación productiva, la empresa generó la capacidad necesaria para brindar servicios y condiciones que a priori permiten una sustancial reducción de costos a nuevos productores. En este sentido, por un lado los nuevos productores de pacú de la región contarán con un laboratorio de alevines capaz de generar larvas y alevines en origen, evitando así costos asociados al flete y reduciendo los riesgos propios del traslado de un insumo de tal vulnerabilidad. En efecto, actualmente el laboratorio de alevines entrega de forma gratuita ejemplares a productores interesados de la zona, para que realicen experimentaciones y pruebas piloto. Por otro lado, también podrán acceder a la alimentación suplementaria en el mercado local gracias a la planta procesadora de alimento balanceado de peces. Esto representa una ventaja de gran relevancia considerando el significativo peso de la alimentación de los peces en los costos totales de la actividad (70\%). Tal peso sería aún mayor si los alimentos se adquirieran fuera de la zona por el respectivo incremento de los costos de transporte. De esta manera, los seguidores que surjan podrán no sólo procesar el pescado en el frigorífico local, sino además encontrar canales de comercialización ya generados o incluso comercializarlos bajo la marca existente (Teko). 
Para los productores de arroz, los costos también se reducirían considerablemente, en especial en el eslabón comercial, dado que la presencia del molino arrocero cercano al futuro muelle permitirá acopiar la producción en origen y trasladarla por vía fluvial. De esta manera, se reducirá el costo del flete volviendo más rentable la actividad.

En síntesis, el caso presentado permitió identificar diferentes vías a través de las cuales el desarrollo de la empresa pionera reduce potencialmente las barreras a la entrada para nuevas empresas, tanto al disminuir los costos de aprendizaje de los ingresantes como al proveerlos de insumos y servicios complementarios que tornan rentables emprendimientos de menor escala e integración. No obstante, aún no se observan casos concretos de seguidores, lo que abre nuevas preguntas de investigación como la referida al lapso de tiempo necesario para la aparición de seguidores tras el surgimiento de un pionero, o si el surgimiento de seguidores queda restringido por efectos que contrarresten la reducción de barreras a la entrada, como posiciones monopólicas o monopsónicas que podría detentar el pionero.

\section{Referencias bibliográficas}

Artopoulos, A., Friel, D. y Hallak, J. C. (2013). Export emergence of differentiated goods from developing countries: Export pioneers and business practices in Argentina. J. Dev. Econ., 105, 19-35.

Breschi, S., Lissoni, F. y Malerba, F. (2003). Knowledge-relatedness in firm technological diversification. Res. Policy, 32, 69-87.

Cantner, U. y Hanusch, H. (2005). Heterogeneity and evolutionary change - concepts and measurement. En K. Dopfer (Ed.), Economics, Evolution And the State: The Governance of Complexity. Cheltenham, Reino Unido: Google Books.

Cohendet, P. y Llerena, P. (2003). Routines and incentives: the role of communities in the firm. Ind. Corp. Change, 12, 271-297.

David, P. A. y Rosenbloom, J. L. (1990). Marshallian factor market externalities and the dynamics of industrial localization. J. Urban Econ., 28, 349-370.

Dosi, G., Freeman, C., Nelson, R. R., Silverberg, G. y Soete, L. (1988). Technical change and economic theory. Pinter Publishers.

Dosi, G. y Marengo, L. (2000). Some elements of an evolutionary theory of organizational competences. Theory Firm Crit. Perspect. Bus. Manag., 4, 50.

Dosi, G. y Teece, D. J. (1998). Organizational Competencies and the Boundaries of the Firm. En: Arena, R. y Longhi, C. (Eds.), Markets and Organization. Berlin, Alemania: Springer.

Fagerberg, J. (2000). Technological progress, structural change and productivity growth: a comparative study. Struct. Change Econ. Dyn., 11, 393-411.

Glaser, B. y Strauss, A. (1967). El desarrollo de la teoría fundada. Chicago, EE.UU.: Aldine. 
Hausmann, R. y Hidalgo, C. (2010). Country Diversification, Product Ubiquity, and Economic Divergence (SSRN Scholarly Paper No. ID 1724722). Rochester, EE.UU: Social Science Research Network, .

Hausmann, R. y Rodrik, D. (2003). Economic development as self-discovery. J. Dev. Econ., 14th Inter-American Seminar on Economics, 72, 603-633.

Jacobs, J. (1961). The death and life of great American cities. Random House LLC.

Mostafa, R. y Klepper, S. (2017). Industrial development through tacit knowledge seeding: Evidence from the Bangladesh garment industry. Management Science, 64(2), 613-632.

Krugman, P. (1991). Increasing Returns and Economic Geography. J. Polit. Econ., 99, 483-99.

Meichtry, M. (2015). Rotación arroz - pacú. Sinergia productiva y ambiental. AGROPOST, 135.

Meichtry, M. E. (2014). Balance hídrico y energético de la rotación arroz-pacu en terminos de rentabilidad potencial por su eventual sinergia (Tesina de grado). Facultad de Ciencias Agrarias, Universidad Nacional del Nordeste, Corrientes, Argentina.

Nelson, R. R. (1991). Why do firms differ, and how does it matter? Strateg. Manag. J., 12, 61-74.

Nelson, R. R., Winter, S. G. (2002). Evolutionary Theorizing in Economics. J. Econ. Perspect., 16, 23-46.

Nelson, R. R., Winter, S. G. (1982). An evolutionary theory of economic change. Harvard University Press.

Penrose, E. T. (1995). The Theory of the Growth of the Firm. Oxford University Press.

Perroux, F. (1950). Economic Space: Theory and Applications. Q. J. Econ., 64, 89104.

Peteraf, M. A. (1993). The cornerstones of competitive advantage: A resource-based view. Strateg. Manag. J., 14, 179-191.

Prebisch, R. (1959). The role of commercial policies in underdeveloped countries. Am. Econ. Rev., 49, 215-273.

Rumelt, R. P. (1982). Diversification strategy and profitability. Strateg. Manag. J., 3, 359-369.

Sabel, C., Fernandez-Arias, E., Hausmann, R., Rodriguez-Clare, A., Stein, E. (2012). Export Pioneers in Latin America (SSRN Scholarly Paper No. ID 2080388). Rochester, EE.UU.: Social Science Research Network.

Saviotti, P. P. y Frenken, K. (2008). Export variety and the economic performance of countries. J. Evol. Econ., 18, 201-218. 
Teece, D. y Pisano, G. (1994). The Dynamic Capabilities of Firms: an Introduction. Ind. Corp. Change, 3, 537-556.

Teece, D. J., Pisano, G. y Shuen, A. (1997). Dynamic Capabilities and Strategic Management. Strateg. Manag. J., 18, 509-533.

Teece, D. J., Rumelt, R., Dosi, G. y Winter, S. (1994). Understanding corporate coherence: Theory and evidence. J. Econ. Behav. Organ., 23, 1-30.

Wernerfelt, B. (1984). A resource-based view of the firm. Strateg. Manag. J., 5, $171-$ 180.

Winter, S. G. (2003). Understanding dynamic capabilities. Strateg. Manag. J., 24, 991-995.

Yin, R. K. (1994). Discovering the future of the case study method in evaluation research. Eval. Pract., 15, 283-290.

\section{Notas de diario}

Diario La Nación. (01/08/1998).

Diario La Nación. (11/12/2010).

Diario Norte. (09/02/2011).

Diario Norte. (19/03/2011).

Diario Página/12. (14/06/2010).

Diario Página/12. (17/08/2010).

Diario Página/12. (18/03/2011). 